\title{
INTEGRATING SCALE AND SPACE IN 3D CITY MODELS
}

\author{
Jantien Stoter $^{1,2}$, Hugo Ledoux ${ }^{1}$, Martijn Meijers ${ }^{1}$, Ken Arroyo Ohori ${ }^{1}$ \\ ${ }^{1}$ OTB, TU Delft, The Netherlands, \{j.e.stoter |h.ledoux| b.m.meijers | g.a.k.arroy oohori\} @ tudelft.nl \\ ${ }^{2}$ Product and Process Innovation, Kadaster, Apeldoorn, The Netherlands, jantien.stoter@kadaster.nl
}

\begin{abstract}
This paper presents the problem of the current separate treatment of levels of detail in city models. We propose a solution, detail the main principles, and present our initial results on the approach. We conclude with work in progress and explain the benefits of our approach.
\end{abstract}

KEY WORDS: 3D, LOD, scale, generalisation

\section{INTRODUCTION}

CityGML, the OGC standard for modelling and exchanging 3D city and landscape models (OGC, 2008), implements the level of detail (LOD) concept in a separated approach (with 5 LODs from the terrain to the interior of build ings, including furniture), i.e. the different LODs of 3D city models coexist and individual objects are not explicitly linked together. For the storage, maintenance and analysis of these models, this is not optimal and has several limitations.

First, it is particularly difficult to query through different LODs and to keep different LODs consistent after updating. Second, the accuracy measures and structural complexity as described in OGC (2008) for each LOD ("lower than LOD1" in LOD0; $5 \mathrm{~m}$ in LOD1; $2 \mathrm{~m}$ in LOD2; $0.5 \mathrm{~m}$ in LOD3; and $0.2 \mathrm{~m}$ in LOD4) do not work in this differentiated manner in practice: many $3 \mathrm{D}$ models in LOD1 are created from high-accuracy data (e.g. $0.5 \mathrm{~m}$ ) and block models of buildings (LOD1) may have LOD2 semantics attached (i.e. roof and walls). Third, the different LODs refer to individual objects only, i.e. aggregation is not supported and higher LODs cannot consist of parts from a lower LOD. Related to this problem is the lack of a notion of semantic change at a scale transition, for example the concept that single trees at a higher LOD may change to forest at a lower LOD is not supported.

To solve the first two problems, we propose integrating the different LODs of a 3D city model into one consistent fourdimensional data model. In this $4 \mathrm{D}$ hypercube, scale is treated as another dimension perpendicular to the three spatial dimensions. The availability of a $4 \mathrm{D}$ cube that integrates scale and space at a fundamental level offers the possibility to define semantic aspects of scale in the structure in a second step. This provides better ways to manage the scale concept in city models in an integrated manner, and offers a solution for the separate treatment of LODs in CityGML. Therefore the 4D approach provides solutions for the last two problems mentioned above.

While the integration of space and scale has been discussed before, we are currently implementing the concept with a higher-dimensional data structure / data model and working on algorithms to populate the $4 \mathrm{D}$ hypercube. This offers the possibility to continuously zoom-in and out across levels of detail, without jumping to another representation (as in CityGML) because the LODs are integrated in the 4D data structure itself. The actual implementation is the main innovation of this research. In this paper we present our initial ideas and work in progress.
We present in Section 2 a summary of our work. We conclude in Section 3 with some concrete examples where the benefits of our proposed integration of 3D space and scale are highlighted; this also includes tackling the semantic changes that can arise at scale steps by adding semantic knowled ge to the hypercube.

\section{A 4D DATA S TRUCTURE}

The integration of the 3D space and 1D scale into a 4D hypercube creates a representation where no gaps and overlaps may be present. This approach is an extension and a generalisation of the work on variable-scale geo-information, where the different scales/LODs for 2D maps (for example a land use map) are integrated into a 3D structure and stored using a 3D data model (Meijers, 2011).

This contrasts with an approach where mono-scale data sets are independently stored at multiple, but fixed, scale points. The integrated $\mathrm{nD}$ approach aims at reducing redundancy to improve efficiency and to assure better consistency between different scales. Consistency means that the availability of data at different scales is free from contradictions, and this enables smoother zooming in and out.

Figure 1 shows examples of such integration for 2D maps and 1D scale in a 3D structure. It shows that when starting from a $3 \mathrm{D}$ model that is a space partition, a $2 \mathrm{D}$ derived map is again a consistent partition in which all representations fit without any gaps or overlaps.

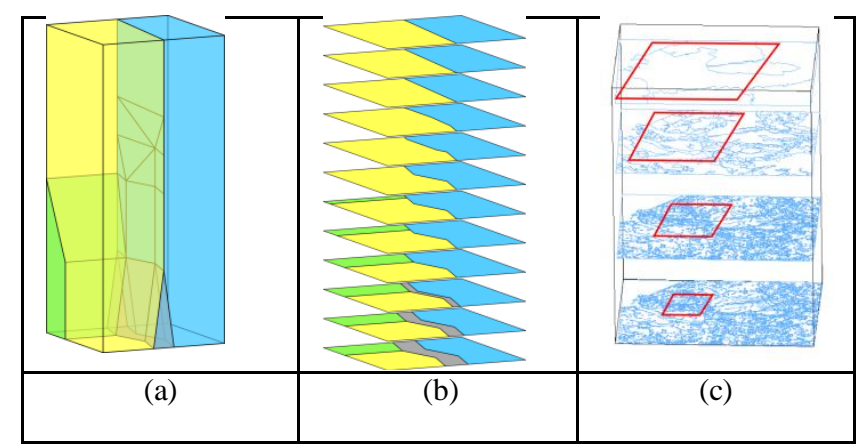

Figure 1. Examples of integrating the 1D scale dimension and 2D space into a 3D structure. (a) Every map object, 4 in this case, is represented as one polyhedron. (b) Maps are slices (cross sections) of this 3D model. (c) For interactive use, apart from taking a slice, also a bounding box filter should be applied. 
Current data models and data structures in GIS are limited to 3D, and higher-dimensional data models are often only theoretical and have not been implemented and used in practice (or only for grids and point data). This is despite the fact that conceptually and theoretically, the generalization of these concepts to one dimension higher, i.e. 3D space plus 1D scale, leading to a $4 \mathrm{D}$ model, is relatively straightforward. We are currently investigating higher-dimensional representations developed in other fields, for instance in computer graphics and computer vision, CAD/CAM, mathematics and topology. The approaches from GIS and combinatorial topology can be considered as opposite and mutually complementary. GIS uses mostly a top-down approach, building data models that are specifically designed for certain applications. These models are thus meant to support the particular operations required in it. In contrast, the bottom-up approach adopted in the fields previously mentioned has been focused on generic mathematical models valid in any dimension, having a solid mathematical background and well-known advantages, e.g. explicit storage of topology, no redundancy, and better quality guarantees under updates.

In our research we attempt to bridge this gap in order to allow higher dimensional modelling in GIS, and apply this to implement scale in city models. We have already identified the generalized maps of Lienhardt (1994), also known as G-maps, as a candidate data model to store $4 \mathrm{D}$ objects and perform operations on them. Other alternatives, such as the cell-tuple structure of Brisson (1989), could also have been used, but Gmaps has the advantage of having been implemented in 3D (it is used in GOCAD ${ }^{1}$ for geological modelling and in $\mathrm{Moka}^{2}$ for geometric modelling), and of being proven to be able to represent a wider class of objects.

We are currently studying the necessary modifications to mathematical data models in order to implement a higherdimensional GIS. These modifications include support for geometry at the point level, holes, spatial indexing, easy visualisation, and disconnected embeddings. These modifications are explained in more detail in Arroyo Ohori et al. (2012).

It should be noted that while G-Maps and other similar structures have been implemented in a $4 \mathrm{D}$ context, they use special operations to merely link a series of fixed LODs (Fradin, 2002). Our integration is different since it uses all dimensions in the same manner, treating all dimensions as if they were spatial.

By doing so, were are able to create operations that operate on multiple dimensions simultaneously, such as mixed scale slicing (Figure 2), similarity and 4D distance computations, spatiotemporal validation, etc.

To build the hypercube, we plan to start with LOD3 models and perform automatic generalisation to obtain the LOD2 and the LOD1; algorithms such as the ones of Guercke and Brenner (2009) and Zhu (2010) can be used. Since we control the generalisation process, it will be possible to build the hypercube in an incremental manner (and thus ensure its consistency).

\section{THE BENEFITS OF THE INTEGRATION}

The hypercube results in a scale-less, continuous representation of a city model, i.e. not restricted to 5 fixed LODs (in the case

\footnotetext{
${ }^{1}$ http://www.gocad.org/

${ }^{2} \mathrm{http}: / /$ moka-modeller.sourceforge.net/
}

of CityGML for instance). Slicing this hypercube permits us to obtain a city model at a chosen LOD.

The integrated cube has potentials that are not possible by simply linking LODs.

This can be compared with the two main approaches that exist for maintaining $2 \mathrm{D}$ data sets at different scales. The first option is to separately maintain different databases at predefined scale levels and requires linking LODs to assure consistency. The second option is to maintain only the most detailed data and to automatically generalize it to obtain the small-scale data, often pre-storing the results of costly geometric computations in multi-representation (as in the first option). This second option is similar to our 4D approach.

Since automated generalistion has been very complicated until recently, the first option (maintaining 2D datasets at different scales) is practiced by many National Mapping Agencies that produce maps at different scales. Many researchers have studied 2D multi-representation data models (that can also be used to link different 2D LODs) since it was introduced by NCGIA (1989) and Buttenfield et al. (1998). Examples are MRMS (Friis-Christensen and Jensen, 2003), MADS (Parent et al, 2006), Perceptory (Bedard et al, 2004), Modelling multiple geometries (Jones, 1996), modelling scale transitions between pairs of objects (Devogele, 1996); modeling links between instances (Kilpelainen, 1997) and modeling a multi-scale data model (Stoter at al, 2011). While these initiatives (in 2D) aimed at controlling the redundancy of multi-representations and multi-scale data, the $\mathrm{nD}$ approach in our research aims at reducing redundancy (as in the second option) to improve efficiency and to better assure consistency between different scales. This is the main difference between linking different LODs to manage sale in city models and our 4D approach.

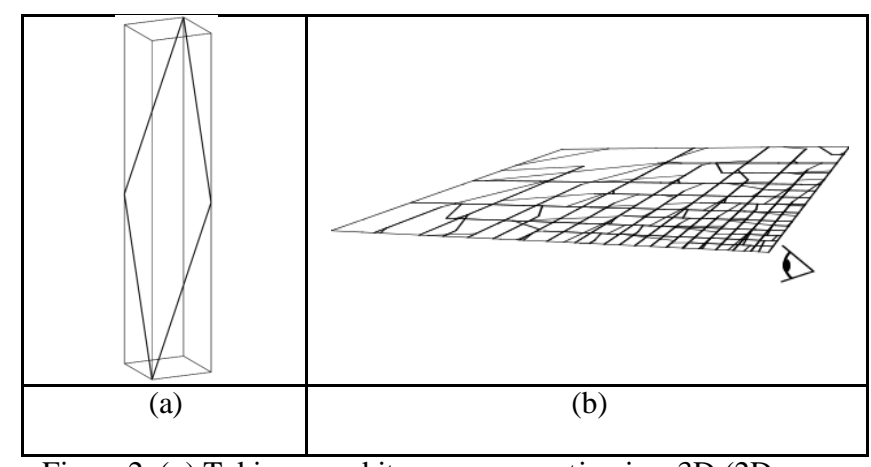

Figure 2. (a) Taking an arbitrary cross section in a 3D (2D space $+1 \mathrm{D}$ scale) cube leads to (b) a derived 2D representation that has mixed scale: close to the observer much detail is shown, while further away less detail is obtained. For 4D models (3D space + 1D scale), a similar operation can be performed.

The data structure solves the integration of scale and space at a fundamental level. The next step is to add semantic knowledge to tackle semantic changes at scale steps and model these semantic changes via de $4 \mathrm{D}$ data structure. This requires the incorporation of operations that enable contextual generalisation of 3D data and will enrich the 4D data structure with semantic concepts. For example aggregation of single buildings when going from LOD2 to LOD1, which is an operation studied in Guercke and Brenner (2009) and Zhu (2010). To further support the semantic concepts of scale chan ges, we also plan to perform constrained generalisation. That is, if different LODs are available, we generalise between them but ensure that the resulting object at a given LOD is the same as the existing object at that LOD. This lays down an explicit link between existing LODs (also studied in the work of Bédard, Y. \& E. 
Bernier (2002)). This is a generalisation to 3D of work previously done in 2D (Dilo et al., 2009).

Examples of applications where an integrated approach is useful are noise and wind simulation.

Simulations are rather complex and need city models as input. However, for performing a simulation efficiently more details is required close to the object under study, while far away a coarse model will often be sufficient. With the method we propose, it boils down to slicing in a particular way (e.g. for 2D+1D using a bell-shaped surface), and this generalizes into using a hypervolume for the $4 \mathrm{D}$ case, obtaining 3D data with the appropriate amount of detail. The intersection of this 4D hypercube with the hypervolume gives a perfect 3D topology: all representations fit without gap s or overlaps. Figure 3 shows an example of noise modelling that could benefit from having more details near the railway.

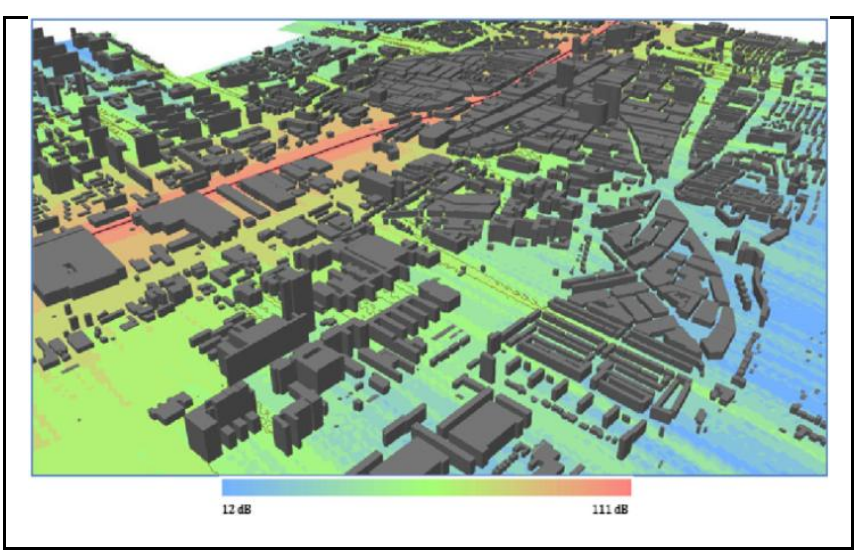

Figure 3. Noise modelling in 3D (caused by a railway in downtown Delft) would benefit from having more detail available close to the source of the noise, while further away less detail is needed.

\section{ACKNOWLEDGEMENTS}

This research is supported by the Dutch Technology Foundation STW, which is part of the Netherlands Organisation for Scientific Research (NWO) and partly funded by the Ministry of Economic Affairs, Agriculture and Innovation (project code: 11300 and 11185).

\section{References}

Arroyo Ohori, K., Ledoux, H. and Stoter, J. 2012. HigherDimensional Modelling of GIS Datasets with the G-maps Data Structure. Submitted to SDH 2012.

Bédard, Y. \& E. Bernier, 2002. Supporting Multiple Representations with Spatial View Management and the Concept of "VUEL". Joint Workshop on Multi-Scale Representations of Spatial Data, ISPRS WG IV/3, ICA Com. on Map Generalization, July, 7-8, Ottawa, Canada.

Bédard, Y, S. Larrivée S, M-J Proulx, M. Nadeau. 2004. Modelling geospatial databases with plug-ins for visual languages: a pragmatic approach and the impacts of 16 years of research and experimentations on Perceptory. Berlin : Springer, 2004. LNCS 3289

Brisson, E., 1989. Representing geometric structures in d dimensions: topology and order. In Proceedings of the 5th annual symposium on Computational geometry, pages 218-227, New York, NY, USA, 1989. ACM.

Buttenfield, B.P. and J.S. Delotto. 1989. Multiple representations. Scientific Report for the Specialist Meeting. National Center for Geographic Information and Analysis (NCGIA), 1989. p. 87. Technical paper 89-3.

Devogele T., J. Trevisan and L. Raynal. 1996. Building a multiscale database with scale transition relationships. International Symposium on Spatial Data Handling. pp. pp 337-351.

Dilo, A. and P. van Oosterom and A. Hofman, 2009. Constrained tGAP for generalization between scales: The case of Dutch topographic data. Computers, Environment and Urban Systems, Volume 33(5), pp. 388-402.

Emgård, K.L. and S. Zlatanova, 2008. Design of an integrated 3D information model. [red.] Rumor, Fendel and Zlatanova Coors. London, UK: Taylor \& Francis Group. Urban and regional data management: UDMS annual 2008. pp. 143-156.

Fradin, D., Meneveaux, D. and Lienhardt, P., 2002. Partition de l'espace et hiérarchie de cartes généralisées: application aux complexes architecturaux. Actes des XVèmes journées de l'Association Française d'Informatique Graphique, Volume 28(2).

Friis-Christensen, C.S. and A. Jensen. 2003. Object-relational management of multiply represented geographic entities. Cambridge, MA, USA, July 9-11, 2003. Proceedings of the Fifteenth International Conference on Scientific and Statistical Database Management.

Guercke, R. and C. Brenner, 2009. A Framework for the Generalization of 3D City Models. Proceedings 12th AGILE International Conference on Geographic Information Science, Hannover, Germany.

Guercke, R. and T. Götzelmann, C. Brenner, M. Sester, 2011. Aggregation of LoD 1 building models as an optimization problem, ISPRS Journal of Photogrammetry and Remote Sensing, Volume 66, Issue 2, March 2011, Pages 209-222.

Jones C.B., Kidner D.B., Luo L.Q., Bundy G.L., Ware J.M. 1996. Database design for a multi-scale spatial information system. International Journal Geographic Information Science, Vol. 10, pp. 901-920.

Kilpelainen, T. 1997. Multiple representation and generalisation of geo-databases for topographic maps. PhD thesis. Finnish Geodetic Institute, 1997.

Lienhardt, P., 1994. N-dimensional generalized combinatorial maps and cellular quasi-manifolds. International Journal of Computational Geometry and Applications, 4(3):275-324.

Meijers, M., 2011. Variable-scale Geo-information. PhD thesis, Delft University of Technology. Netherlands Geodetic Commission.

NCGIA. National Center for Geographic Information and Analysis, 1989. The research plan of the National Center for Geographic Information and Analysis. International Journal Geographical Information Systems, Vol. 3, pp. 117-136. 
Parent, C, S. Spaccapietra and E. Zimányi. 2006. Conceptual modelling for traditional and spatio-temporal applications. The MADS approach. Springer, 2006. ISBN: 3-540-30153-4.

Jantien E. Stoter, Thomas Visser, Peter van Oosterom, Wilko Quak, Nico Bakker: A semantic-rich multi-scale information model for topography. International Journal of Geographical Information Science 25(5): 739-763 (2011)

Zhu, Q., Junqiao Zhaoa, Zhiqiang Dua, Yeting Zhanga, 2010, Quantitative analysis of discrete 3D geometrical detail levels based on perceptual metric, Computers \& Graphics

Volume 34, Issue 1, February 2010, Pages 55-65 